\title{
An "EPIIC" Vision to Evolve Project Integration, Innovation, and Collaboration with Broad Impact for How NASA Executes Complex Projects
}

\author{
Robert J. Moreland \\ National Aeronautics and Space Administration \\ 300 E. Street S.W. \\ Washington D. C. 20546 \\ 202-329-8851 \\ RobertMoreland@nasa.gov \\ John W. Evans \\ National Aeronautics and Space \\ Administration \\ 300 E. Street S.W. \\ Washington D. C. 20546 \\ 202-358-0937 \\ John.W.Evans@nasa.gov \\ Jessica Knizhnik \\ National Aeronautics and Space \\ Administration \\ 8800 Greenbelt Road. \\ Greenbelt, MD 20771 \\ 301-286-5728 \\ jessica.knizhnik@nasa.gov \\ Anna-Maria R. McGowan \\ Nipa Phojanamongkolkij \\ National Aeronautics and Space \\ Administration \\ 17 West Taylor St. \\ Hampton, VA 23681 \\ 757-864-2846 \\ anna-maria.r.mcgowan@nasa.gov \\ National Aeronautics and Space \\ Administration \\ 17 West Taylor St. \\ Hampton, VA 23681 \\ 757-864-6396 \\ nipa.phojanamongkolki@nasa.gov \\ Prepared by the National Aeronautics and Space Administration. \\ No copyright is claimed in the United States under Title 17, U.S. Code. Published and used by INCOSE with permission.
}

\begin{abstract}
Evolving Project Integration, Innovation, and Collaboration (EPIIC) is a vision defined to transform the way projects manage information to support real-time decisions, capture best practices and lessons learned, perform assessments, and manage risk across a portfolio of projects. The foundational project management needs for data and information will be revolutionized through innovations on how we manage and access that data, implement configuration control, and certify compliance. The embedded intelligence of new interactive data interfaces integrate technical and programmatic data such that near real time analytics can be accomplished to more efficiently and accurately complete systems engineering and project management tasks. The system-wide data analytics that are integrated into customized data interfaces allows the growing team of engineers and managers required to develop and implement major NASA missions the ability to access authoritative source(s) of system information while greatly reducing the labor required to complete system assessments. This would allow, for example, much of what is accomplished in a scheduled design review to take place as needed, between any team members, at any time. An intelligent data interface that rigorously integrates systems engineering and project management information in near real time can provide substantially greater insight for systems engineers, project managers, and the large diverse teams required to complete a complex project. System engineers, programmatic personnel (those who focus on cost, schedule, and risk), the technical engineering disciplines, and project management can realize immediate benefit from the shared vision described herein. Implementation of the vision also enables significant improvements in the performance of the engineered system being developed.
\end{abstract}




\section{Introduction}

The complexity of spacecraft systems continues to grow as measured by a variety of metrics surveyed in the literature (Lin, 2011; Leising, 2013; Dvorak, 2009). While there is no consistent complexity metric, whether we consider the number of instruments, design complexity, project programmatic complexity, or the growth in size and complexity of flight software, the aerospace community is generally aware of increasing project complexity with a heightened awareness of complex interdependencies.

While complex interdependencies draw attention to cross-system connections, the sheer volume and complexity of interdependencies can also foster a hierarchical or siloed system view to manage information and simplify understanding. No longer can any one person (nor small team of leaders) intuit all there is to know about a single large aerospace system. The availability and diversity of data about the engineered system is escalating at a rate faster than many program and project management systems can handle. The increased complexity and availability of data often exists in an outdated organizational and operational environment that relies on sharing and updating information in a manner ill-suited for the system dynamics described above. For example, project data is often exchanged via the frequent use of emails; and, configuration control is often handled by documents. Rather than real-time integration, project data and configuration control are typically integrated during design review events, with subsequent time-consuming actions to reconcile disconnects. A hierarchical or siloed systems view also fosters more localized risk-management rather than addressing challenges from a system-wide view.

This paper articulates a vision for evolving the way NASA manages information for highly complex projects. The authors have named this vision "Evolving Project Integration, Innovation \& Collaboration" (EPIIC). EPIIC embraces the combined impacts of emerging digital technologies and data analytic capabilities to enable improved system integration. From an institutional perspective, EPIIC is transformative, yet, as the name implies, EPIIC can intentionally be implemented in an evolutionary manner: initial integration first across disciplines supporting a project throughout the project lifecycle; then across multiple projects; and eventually throughout the entire mission/program/project life-cycle including the research, technology development, and operations phases.

\section{Background: Managing Complex Information for NASA Projects}

Figure 1 is used to show the current challenge in managing information on a complex project. Each function maintains their own functional data system (e.g., local computers, shared network drives within the organization, Web Content Management (WCM) system, etc.), which is depicted by a disc for each function. As the project progresses through its life cycle, each function creates or updates their models, designs, and documents locally even though these items are intended to be aligned across all functions. In a preparation for a project's gate review, each function will upload their latest version onto the project's centralized Technical Data Management System (TDMS), which is represented by the yellow disc in the center and is a secured, data and configuration managed WCM system used mainly as the project's repository for archival purposes.

After the successful completion of each gate review, the TDMS's functional documents will be configuration controlled as required by the project management procedural requirements. If the latest version is in its native model format (e.g., project's integrated master schedule, 3D drawings of the systems, etc.), there will be an effort to convert the native format into the document format. One example is taking screenshot images of the model and pasting them onto the document, with a signature approval page as the cover page. Once all approvers manually sign, the signed signature page will be scanned and attached electronically on the project's TDMS. 
In addition to the configuration-controlle d copies in the project's TDMS, contents on the functional data systems usually include models for exploration purposes and all uncontrolled interim document versions. Any collaborative cross-discipline analyses between the gate reviews are done through email exchanges, phone conversations, or meetings to request or track discipline-related information necessary for the cross-discipline analyses. This type of collaboration is merely coordination, with time spent not only on verifying inconsistent information (e.g., individual discipline lexicons, unit of measures, etc.), but also on verifying the latest version of information. Analyses emerging from different disciplines may not reflect the most recent design changes occurring on the project as result of ineffective coordination across large complex projects with scores or even hundreds of personnel providing data from geographically-dispersed locations. These coordination activities, which are focused on data collection and preparation, are depicted by the linkages among functions in Figure 1, shown as dashed lines. There are other collaborative and innovative activities (the solid lines) that exist as well. These thin lines represent team collaborations that are invaluable for problem solving, brainstorming, or creating alternative solutions. Unfortunately, the network portrayed in Figure 1 is often dominated by data collection and preparation activities. This network becomes increasingly (and even unmanageably) intricate as project, team, and information complexity grows.

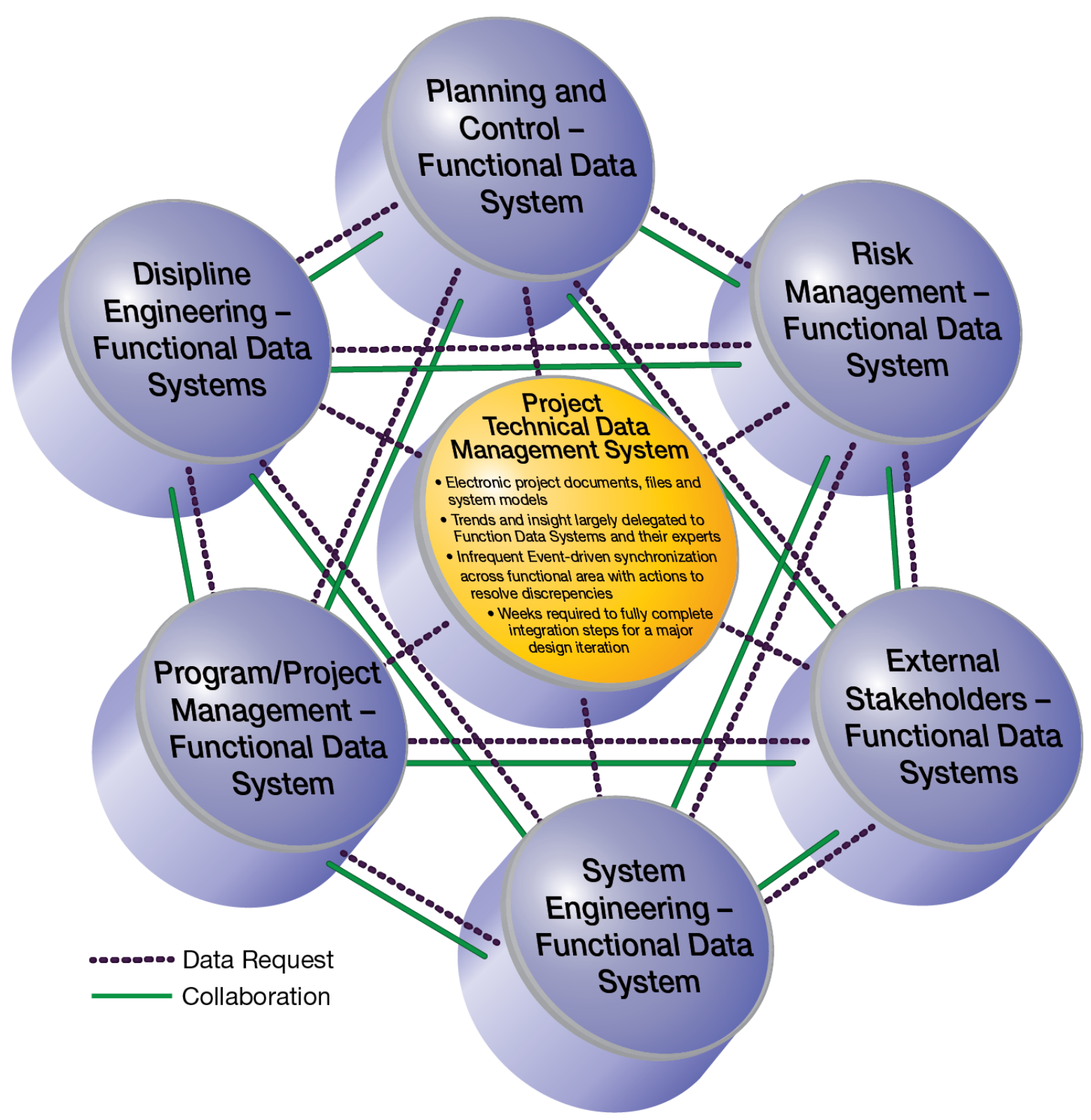

Figure 1. Current functional network for project management 


\section{The EPIIC Vision}

The EPIIC vision is described in a series of graphics to represent the implementation of the vision first described across a single project over its project lifecycle, then across multiple projects, then across the entire lifecycle of activities required to support NASA missions, programs, and projects. The entire lifecycle for NASA missions/programs/projects such as human exploration or planetary science includes consideration of basic research and technology maturation all the way through operations and assessments of performance and reliability compared to plans. The following Figures 2, 3, and 4 are, progressively, EPIIC's evolution from today's approach shown in Figure 1, with growing benefits as the vision is applied more broadly.

\section{The EPIIC Vision Implemented Across a Single Project}

Foundational to EPIIC's vision are two key concepts:

1) A single, fully integrated, digital authoritative source of information that is always up to date

2) A digital data management system that has embedded intelligence based upon digital transformation that uses model-based, data-analytic, and machine-learning approaches to provide comprehensive and near-real time integration of data

There are several motivations for the above concepts. Today, project subject matter experts (SMEs) spend considerable time requesting different types of data then manually integrating the new data with what they already have. Utilizing enhanced model-based and data-analytic approaches to automatically integrate data and update analyses allows SMEs to focus on technical assessment, collaboration, and improvements. Automating much of the integration activity, including subsystem models and interfaces, enables higher fidelity optimizations and integration typically considered impractical for large complex projects.

The EPIIC vision also seeks to transform design iterations. Where normally several weeks are required to complete the full assessment of all integration steps for a major design iteration, using the embedded intelligence envisioned, a design iteration can shrink to days for the full integration depending on the level of information and analytical capabilities available. As we expect information/data-analytic and computational capabilities will continue to rapidly advance in the coming years, several benefits will result, (a) system integration can potentially drop to near-real time, and (b) with each design iteration's increasing detail and specificity over the project lifecycle, higher resolution artifacts and project views are possible integrating previously disparate data sources for improved decision making, monitoring the project's performance and trends.

The vision for a project's future collaborative functional network is illustrated in Figure 2. Similar to the current functional network in Figure 1, each function has their own functional data system (FDS), which contains uncontrolled and explorative-in-nature models or documents uniquely pertaining to its function. The key difference illustrated in Figure 2 is the common availability of the project's centralized Digital Data Management and Analytics (DDMA) that contains all information necessary for all functions to exchange, interact, and collaborate digitally with a true source of project data. This information can be determined by the $\mathrm{N}$-Squared ${ }^{1}$ diagram technique (NASA Systems Engineering Handbook). The DDMA can be accessed interactively serving as a repository of the configuration controlled information as well as the latest project's information. The DDMA now evolves to include embedded intelligence, relying upon statistical engineering, machine learning and data analytics, etc. to identify and anticipate trends within the project, and, as we see later in Figure 3 , these capabilities can be extended to work across multiple projects as well. In summary, the initial

\footnotetext{
${ }^{1} \mathrm{~N}$-Squared diagramis a functional analy sis technique in a matrix representation and is used to identify interactions or interfaces between functions froma systems perspective.
} 
implementation of the EPIIC vision (described in Figure 2) is focused within a single project over is project lifecycle, where intelligent DDMA will facilitate, for example: automated workflow management, cross-discipline change assessments and management, interactive programmatic and technical health assessments, model-based certifications, and automatically generated gate review charts on-demand.

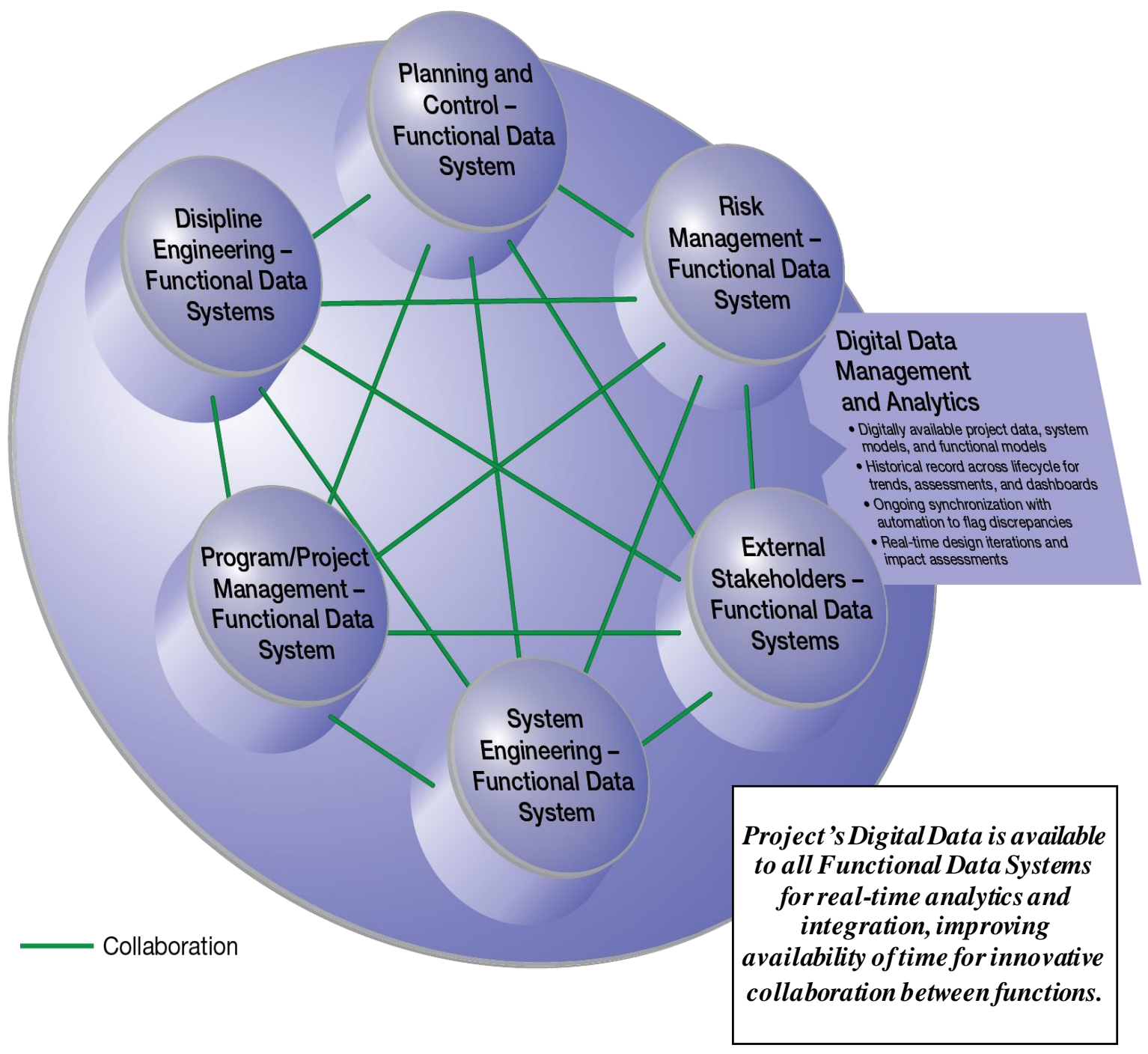

Figure 2. EPIIC vision across a single project

It is important to note that the DDMA's contents will be input and output information for all interacting functions which can be determined using an N-Squared diagram technique. The FDS for each function will contain any information not required by the project in the DDMA. In other words, the DDMA contains all the project data required for a major design review, while the FDSs contain the institutional knowledge about the function's models, lessons learned, and historical data sets across many projects. Given project information is readily available in the DDMA, there is no need to make or track data requests, verify inconsistent information, or confirm the latest version of information. By reducing the overhead of these activities, project team collaboration activities can focus on innovation, risk reduction, and improved system performance.

Potential benefits of implementing the EPIIC vision across a single project include:

(1) Efficient processes with less time collecting data, requesting data, and reconciling inconsistent data, which results in savings to the programmatic/cost/schedule/external stakeholder community for transparent data/information access;

(2) Effective processes while minimizing analysis errors due to outdated data, and its inconsistencies; 
(3) Single (and reusable) source of data which is export, version, and configuration controlled;

(4) Enabling increased attention to innovative design solutions and other technical needs to enhance the engineered systems performance.

\section{Some Technologies and Capabilities to Enable the EPIIC Vision}

In the descriptions below, we offer a few simple examples regarding how some technologies and capabilities enable the EPIIC vision. This discussion is presented to identify the collective value of utilizing and integrating advanced technologies and is not intended to identify the latest research in each topic area.

Model-Based Sys tems Engine ering. The INCOSE SE Vision 2020 (INCOSE, 2007) defines ModelBased Systems Engineering (MBSE) as "the formalized application of modeling to support system requirements, design, analysis, verification and validation activities beginning in the conceptual design phase and continuing throughout development and later life cycle phases. MBSE is part of a long-term trend toward model-centric approaches adopted by other engineering disciplines, including mechanical, electrical and software." Enabling MBSE as a standard practice requires several engineering standards for technical discipline-based lexicons within a portfolio. These standards (referred to here as ontologies) enable model reuse and building a model-of-models. Several examples include (JPL Modeling and Simulation website, 2018; Weiland \& Holladay, 2017; Phojanamongkolkij, 2017; Sundaram \& Brownlow, 2018).

Automation of Information Integration. Computers now have the capacity and speed (Herkewitz, 2015; NASA High-End Computing Capability website, 2018) to integrate models (of higher and higher fidelity) that were previously relegated to disparate systems operated by subject matter experts (SME's). During early project formulation, this will reduce timelines to iteratively optimize designs and enable more tightly integrated solutions, without traditional delays for emailing data requests and other "person-in-the-middle" practical limitations. Objective optimization at the system level potentially enables organizational assignments and solutions to compete on a more technically-s ound basis because the models can accommodate solutions sets that represent many different inputs before assignments are made. For example, competencies vary by organization, such that "propulsion" at one NASA center may include a bias for operational simplicity using pressure-fed hypergolic propellants, and another NASA center may have the strong relationships needed for integration of propulsion and environmental control, leading to a propellant selection that includes oxygen. Optimization of the design depends on not making the organizational assignment too early. And, during operations, the automated integration enables intelligent assistants to automatically provide useful information and advice support to the system architect for a complex Earth observing satellite system for example (Bang, 2018).

Visualization. Using visualization (NASA Information Architecture and Data Management web site, 2018) to display multiple disparate data sources for operations of spaceflight hardware is a direct benefit. Considering how we might also use these same technologies throughout the project development lifecycle provides more leverage for their development. These will include dashboards for programmatic and technical health assessments, cost and schedule visualization and integrated models with 3-D graphics for managing configuration control and project communications.

Digital Authoritative Source for Project Data. Commensurate with using advanced computational capabilities to integrate SME models during formulation, these capabilities can also be used to provide an authoritative source of digital project information, dynamically available and configuration controlled, that offer benefits beyond the traditional document-centric approach to control project data. This improved ability for configuration control of project information being digitally available will lead to a preference for model-centric certification, not our current approach for document-centric certification. Several examples include (Zimmerman, 2017; Singh \& Willcox, 2018; Mavris, 2018). 
Machine learning. One application is to deploy machine learning to study old data for new outcomes (e.g., to identify new planets using machine learning algorithms on existing telescopic records). The new application we hope to illuminate is described here as a less obvious, process focused application, whereby we propose to use machine learning algorithms to improve the way we manage building complex spacecraft. For example, using hardware that has successfully flown before has merit to reduce risk but understanding the subtle differences in design environments and the impact on reliability is an area to explore by relating these and other historical data sets including test, verification and check-out information. In another application of machine learning, we can imagine the potential to translate between ontologies and disciplines to notionally enable drag and drop file manipulations and merger of previously disparate data sets and models. Several NASA projects and case studies can be found in (NASA Information Architecture and Data Management website, 2018 and NASA Data Strategy whitepaper, 2015).

Role of automation for design. Multiple technologies intersect to enable design automation even within complex systems. For example, linking systems models with Computer Aided Design, structural analysis tools, cost analysis tools, machine learning and optimization scripts, and 3D printers could allow an engineer to enter design parameters and specifications into a system model, optimize the system to meet those design specifications using knowledge from previous designs within the appropriate discipline software (like structural or cost analysis software), push that design into Computer Aided Design software, and utilize 3D printing techniques to quickly create a physical prototype. With a well-structured model, design can also be facilitated with automated analysis of reliability and safety to enhance fault tolerance implementation and offset risk. This combined knowledge could allow for unique and more optimal designs than a single human might have envisioned or generated. Further, engineers could focus attention toward making decisions more suited to human intellect such as examining model assumptions in new contexts, and using lessons learned from historic data in new way. Overall, a more complex mission might be undertaken with fewer resources and greater performance.

Intellige nt Requirement Interpre tation. The key to realizing the vision will rely on the ability to interpret requirements based on command and context. Intelligent algorithms will allow computers to navigate uncertainty and learn, correctly establishing operator intent with human-in-the-loop verifications. The sometimes-overwhelming constraints of semantics, ontology, and taxonomy are likely required structure but they become invisible as we develop intelligent algorithms to compare libraries of data and contextual information, allowing the computer to codify accurate relationships between other models and data such as requirements and interfaces. This capability may already be available today as reported in (Burggraf, 2018; Liu, 2018).

\section{Provisional Use Case for Project Management}

Before expanding upon the EPIIC Vision across a Single Project to include a Vision across Multiple Projects, the authors would like to further illuminate the impact of changes represented by Figure 2. While progress has been made in developing tools and experience implementing MBSE, converting old processes to integrate information manually into automated processes, and developing visualization applications, the realization of all these improvements across all stakeholders to include benefits of a Digital Authoritative Source for project data has not yet occurred. New stakeholders are available as early adopters from the programmatic communities for acquisition, cost, schedule, and risk. Considered together and strategically, each improvement opportunity is more compelling by including additional communities who can contribute and benefit from the improved project management vision. One scenario with integrated results is described in Table 1 . 
Table 1: The EPIIC Vision across a Single Project, Provisional Use Case

\begin{tabular}{l}
\hline Current Practice (Fig. 1) \\
\hline For a typical document-based approach, subject \\
matter experts (SMEs) upload their models or \\
documents onto a technical data management \\
system (TDMS) for archival only. The models and \\
documents can easily become out of sync with each \\
other, and some analyses are based on outdated \\
data. Interdependencies between the discipline \\
models and documents may not be obvious. When \\
a change is proposed, a configuration control board \\
(CCB) assesses the impact. While all SMEs are \\
responsible for identifying and characterizing any \\
new risks, accurate and comprehensive assessment \\
is challenged due to the difficulties in identifying \\
interdependencies between the discipline models \\
and documents.
\end{tabular}

Provisional Future Vision (Fig. 2)

With a Model-Based approach, the data

management, configuration control and risk management processes are tightly coupled.

SMEs will engage a series of interactive webinterface forms to upload their models or documents to the intelligent Digital Data Management and Analytics (DDMA) system(s) and properly associate them. A smart system flags interdependencies, initiates an automated workflow, and requests the owners to verify and approve. Once accepted, a CCB may assess the system impacts and identify any new potential risks. After the CCB approves, the project status's dashboard will be automatically updated. The digital workflow will then remind SME's to update their risk's status. Automated analysis products support the risk assessments.

Many projects assess plans using risk-informed Given all the data needed are readily available, the embedded intelligence of the DDMA can create consistent formats for the data to be displayed and utilized as needed. The DDMA enables the use of data analytics to do near real time integrated calculations, such as the project's Cost and Schedule Confidence Levels and EVM analyses and assessments with substantially less effort to collect, reconcile, and reformat data.

For a project to advance through its lifecycle, all subsystem and system leads prepare gate review packages for specific project integration events, often in PowerPoint format, and unlinked to the actual TDMS. After review, SME's submit Request for Actions (RFAs) or Review Item Discrepancies $(R I D s)$. These RFAs or RIDs are assigned to team members for a response which is often an iterative process before the review is considered completed.

Chief Safety and Mission Assurance Officer (CSO) as independent technical authority monitors project risks and problem reporting through project risk board meetings, documents and emails. Risk acceptance occurs prior launch through final gate review in typical PowerPoint format.

The review packages are generated directly from the WCM on-demand and stakeholders can access actual project data directly. The preparation of review packages is no longer labor intensive (or necessary), and the gate review process is ongoing. Fewer RFA's and RID's are expected, managed when identified instead of waiting for design reviews, essentially eliminating document-based certifications and manual routing workflow.

CSO monitors project through a dashboard linking models, analysis, problem resolutions, project data, and SMA products that can be queried to reveal the project status in abating risk. This becomes the basis for risk acceptance prior to launch, supporting key decision makers with structured information and uncertainty analysis. 


\section{The EPIIC Vision Implemented across Multiple Projects}

The next step in the EPIIC vision is the implementation across multiple projects, depicted in Figure 3. In Figure 3 each layer shown represents a different aerospace project. Here, multiple projects can directly share information, embed lessons learned, and reuse models and heritage hardware. In this vision, each function continues to manage the institutional knowledge about the function's models, lessons learned, and historical data sets across many projects while also now having access to prior complete project data sets at the integrated system level, formerly relegated to documents stored in the TDMS and generally unavailable or difficult to obtain after project completion.

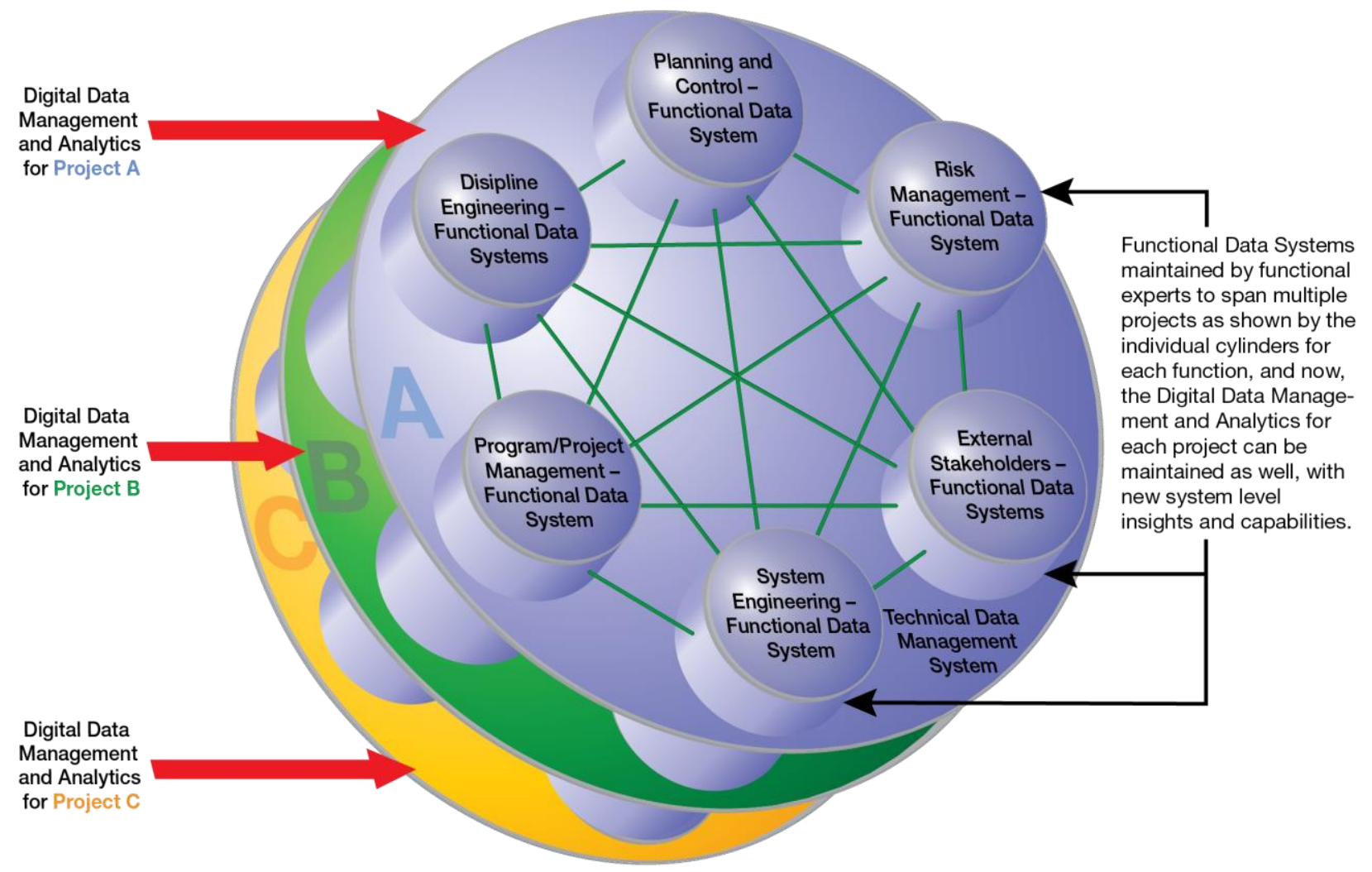

Figure 3. EPIIC vision across multiple projects

There are a number of benefits of implementing EPIIC across multiple projects that include:

(1) Efficient consideration of cost, schedule, and risk informed by prior projects;

(2) Effective processes with knowledge sharing across multiple projects;

(3) Ability to embed lessons learned into models, "hardwiring" the lesson from what is now a document-based repository;

(4) Ability to reuse hardware from one project to another, and capture the peculiarities of each application in the model to ensure heritage hardware is applied within prior contexts;

(5) Effective use of data sources to support safety and reliability analyses across a portfolio of projects enhancing safety across the enterprise.

\section{The EPIIC Vision Implemented across the Entire Lifecycle}

Figure 4 depicts the expectation for collaboration throughout the entire mission/program/project lifecycle including the research and technology development phase, operations, and the application of data collected during operations by the scientists and engineers. In addition to the view across many projects shown in Figure 3, the EPIIC vision in Figure 4 introduces other critical stakeholders with shared objectives for mission/program/project success. These include institutional or matrix engineering organizations investing limited resources to improve technology readiness levels, and in NASA's context, at the other end of the lifecycle are the scientists and academic community who use 
the data from our projects to further their science objectives and the mission operations personnel who operate the spacecraft and develop mitigations when problems emerge.

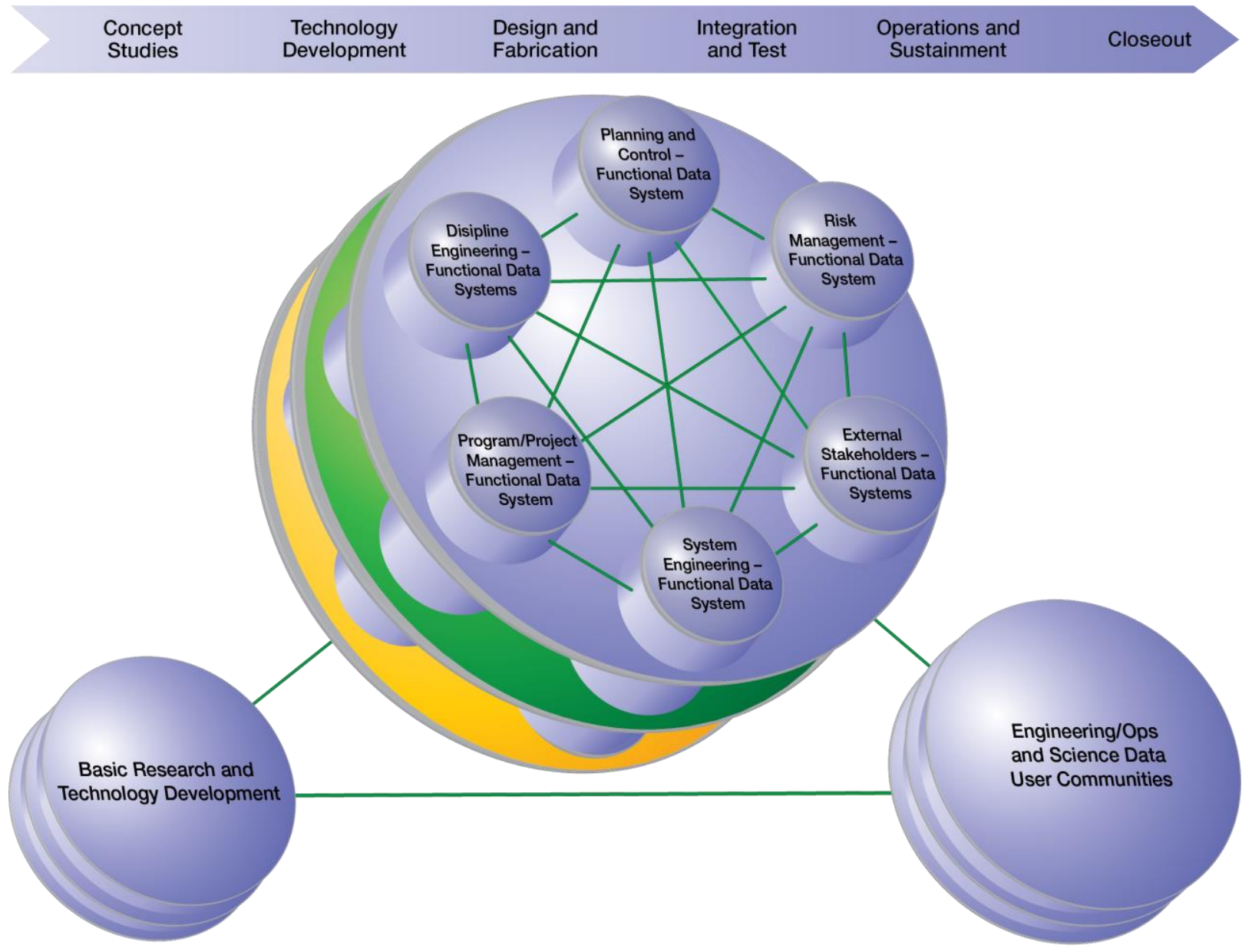

Figure 4. The EPIIC vision implemented across entire lifecycle

The entire lifecycle for NASA activities is broader than any particular project lifecycle and Figure 4 is used to show how basic research and technology development informs what is possible to achieve on future projects. Figure 4 also shows the connection with the Engineering/Ops and Science Data user communities to provide feedback to the project community regarding efficacy of the project to meet its operational objectives, what risks were realized during operations, and actual reliability. Research and technology development collaboration with the project community aligns future abilities and research interests with actual projects and including the Engineering/Ops and Science Data user communities, helps to ensure lessons learned and best practices are identified and disseminated. While the intent for this cradle-to-grave perspective is present today, in practice, the different communities will benefit from more routine access to data across the entire lifecycle.

Potential Benefits:

(1) Ability to codify and confirm linkage of activities within each community with agency missions and strategic objectives;

(2) Ability to identify high leverage and high priority opportunities appreciating the promulgation of best practices across all projects, not through policy and authority, but by embedding for all time into models matured through technology development;

(3) Enhancement of safety, risk characterization, and risk management due to increased availability of data on actual performance and risk realization during operations to inform basic research and technology development. 


\section{Summary and Next Steps}

This paper proposes a vision for managing project information with increasing complexity and interdependency between many functions, not with any single improvement in technology or skills, but by considering many improvements as a continuum to be achieved over time. This is referred to as the Evolving Project Integration, Innovation \& Collaboration (EPIIC) vision. The EPIIC vision includes concepts for sole authoritative data source, model-based certification, machine learning and big data analytics. The EPIIC vision identifies some of the many technologies and capabilities necessary to achieve the vision as represented by a sequence of graphics for near-term, mid-term, and long-term visions addressing the needs of a single project first, then multiple projects, and then a broader consideration of the entire mission/program/project lifecycle. The near-term vision focuses on EPIIC for a single project. The mid-term vision expands from the near-term to collective projects within a program or mission, where projects share lessons learned, standards, and other critical information. Built upon the mid-term vision, the long-term vision extends the EPIC throughout the mission/program/project lifecycle, from the concept studies to operation and closeout.

Our first next step includes the solicitation of feedback and insights from various communities including leadership, project managers, systems engineers, programmatic analysts, discipline engineers, risk managers, universities, and industry. Internally we anticipate the development of a roadmap(s) to identify future progress against objectives with milestones for Evolving Project Integration, Innovation \& Collaboration (EPIIC). This roadmap may include activities designed to align policy/guidance/training/investments to meet the need and opportunity represented by the EPIIC vision. We anticipate that aligning the sometimes disparate communities engaged in the technologies and skills required for the EPIIC vision, will improve the rationale to make investments in those communities; and position themselves better for adoption by the project management community.

\section{References}

Bang, H., Viros, A., Prat, A., and Selva, D., "Daphne: An Intelligent Assistant for Architecting Earth Observing Satellite Systems,” AIAA SciTech Forum, January 2018, Kissimmee, Florida.

Burggraf, P., Wagner, J., and Koke, B., "Ariticial Intelligence in Production Management - A review of the current state of affairs and research trends in academia," 2018 International Conference on Information Management and Processing, 82-88.

Dvorak, D. et al., "NASA Study on Flight Software Complexity." AIAA 2009, DOI: 10.2514/6.20091882.

Herkewitz, W., "Google and NASA Say Their Quantum Computer Finally Works," https://www.popularmechanics.com/technology/gadgets/a18475/google-nasa-d-wavequantum-computer/ December 9, 2015.

International Council on Systems Engineering (INCOSE). 2007, Systems Engineering Vision 2020. INCOSE-TP-2004-004-02.

Jet Propulsion Laboratory Web site: https://scienceandtechnology.jpl.nasa.gov/research/researchtopics-list/communications-computing-software/lifecycle-integrated-modeling-and .

Leising, C., Wessen R., Ellyin, R., Rosenberg, L., and Leising, A., "Spacecraft Complexity Subfactors and Implications on Future Cost Growth." IEEE Aerospace Conference, 2013, DOI: 10.1109/AERO.2013.6497159. 
Lin, C., Nichols, D., Stone, H., Jenkins, S., Bayer, T., and Dvorak, D., "Experiences Deploying MBSE at NASA JPL.” Frontiers in Model-Based Systems Engineering Workshop, April 2011.

Liu, J., Kong, X., Xia, F., Bai, X., Wang, L., Qing, Q., and Lee, I., “Artificial Intelligence in the $21^{\text {st }}$ Century,” IEEE Access, February 2018, DOI 10.1109/ACCESS.2018.2819688.

Mavris, D., Balchanos, M., Pinon, O., and Sung, W., "Towards a Digital Thread-enabled Framework for the Analysis and Design of Intelligent Systems," AIAA SciTech Forum, January 2018, Kissimmee, Florida.

NASA Data Strategy White Paper, https://niam.nasa.gov/wp-content/uploads/2015/10/DataStrategy-2015-03-17-White-Paper-Small.pdf, March 2015.

NASA High-End Computing Capability website: https://www.nas.nasa.gov/hecc/resources/environment.html Accessed April 2018.

NASA Information Architecture and Data Management Web site: https://niam.nasa.gov/projects/. Accessed April 2018.

NASA Information Architecture and Data Management website https://niam.nasa.gov/lab/visualizations/ Accessed April 2018.

NASA Systems Engineering Handbook, NASA SP-2016-6105 Rev2. Website: https://www.nasa.gov/connect/ebooks/nasa-systems-engineering-handbook September 2017.

Phojanamongkolkij, N., Lee, K., Miller, S., Vorndran, K., Vaden, K., Ross, E., Powell, B., and Moses, R., "Modeling to Mars: a NASA Model Based Systems Engineering Pathfinder Effort." AIAA Space 2017, Orlando, FL, September 12-14, 2017.

Singh, V. and Willcox, K., "Engineering Design with Digital Thread," AIAA SciTech Forum, January 2018, Kissimmee, Florida.

Sundaram, V. and Brownlow, L., "MBSE Digital System Model for AF DCGS," AIAA SciTech Forum, January 2018, Kissimmee, Florida.

Weiland, K. and Holladay, J., "NASA Model-Based Systems Engineering Pathfinder 2016 Summary and Path Forward." 2017 Annual INCOSE International Workshop, Los Angeles, CA, January 28-31, 2017.

Zimmerman, P., "DoD Digital Engineering Strategy." 20th Annual NDIA Systems Engineering Conference, Springfield, VA, October 25, 2017. 